\title{
Interrogation of Ultra-weak FBG Array using Double-pulse and Heterodyne Detection
}

\author{
Tao Liu, Feng Wang, Xuping Zhang, Quan Yuan, Jihui Niu, Lin Zhang and Tao Wei
}

\begin{abstract}
A high performance interrogation method for ultra-weak FBG (UWFBG) using double-pulse and heterodyne detection method is proposed. The perturbation along the UWFBG array is located quickly through the use of double-pulsed optical input waveform. Then the perturbation of fiber is quantified precisely by demodulating the phase of differential signals from a heterodyne configuration. The efficiency of measuring the perturbation is improved by more than 20 times than that of using single probe pulse. In comparison with conventional Rayleigh scattering based approach, the proposed method is supreme in signal-to-noise ratio (SNR), approximately $18 \mathrm{~dB}$ higher. The use of differential signaling method can effectively remove the influence from frequency drift of the laser source, making this proposed method capable of measuring low frequency vibration. In our experiment, perturbations with both sinusoidal and triangle waveform were generated to quantitatively evaluate the performance of the proposed method. The minimum detectable fiber length variation is $14.85 \mathrm{~nm}$, and the sensing frequency can be as low as $0.2 \mathrm{~Hz}$.
\end{abstract}

Keywords-Dynamic vibration measurement; Optical fiber interference; Optical fiber sensors; Optical time domain reflectometry; Ultra-weak FBG

\section{INTRODUCTION}

$\mathrm{P}$ HASE-SENSITIVE optical time domain reflectometry ( $\Phi-O T D R)$ is one of the more popular modalities in distributed optical fiber sensing family. Its superior properties include high sensitivity, fast response speed, long sensing distance, etc. [1-4] Over the past decade, research efforts have been made to develop a series of techniques all towards improvement the performance of $\Phi-O T D R$ technology [5-7]. However, the very weak Rayleigh back scattering signal is fundamentally limiting the performance of all $\Phi$-OTDR

This work is supported by the National Natural Science Foundation of China under Grant No. 61627816, 61540017, 61405090 and 61107074, and the Fundamental Research Funds for the Central Universities under Grant No. 021314380087. (Corresponding author: Feng Wang)

T. Liu, F. Wang, X. Zhang, Q. Yuan, and J. Niu are with the Institute of Optical Communication Engineering, College of Engineering and Applied Sciences, Nanjing University, Nanjing, 210093, China. (e-mail: 2282481805@qq.com; wangfeng@nju.edu.cn; xpzhang@nju.edu.cn; 972498858@qq.com; 2282638092@qq.com)

X. Zhang is also with Key Laboratory of Modern Acoustics, Nanjing University, Nanjing 210093, China.

L. Zhang is with the Aston Institute of Photonic Technologies, Aston University, Birmingham, UK, B4 7ET.(e-mail: 1.zhang@aston.ac.uk)

T. Wei is with the Department of Electrical, Computer, and Biomedical Engineering, University of the Rhode Island, Kingston, RI, USA 02881.(e-mail: tao_wei@uri.edu) technologies. Recently, ultra-weak Fiber Bragg grating (UWFBG) array is proposed. A large number of FBGs with ultralow reflectivity are inscribed along an optical fiber with identical nominal wavelength and constant spacing between neighboring FBGs. The UWFBG array has much stronger reflection than the Rayleigh backing scattering, making it a preferred choice for many quasi-distributed and distributed fiber sensing applications. C. Wang, P. Han and L. Ma et al. have demonstrated various sensing network using UWFBG to perform distributed sensing [8-10].

In general, the phase change of reflected lightwave contains the information of the change of fiber. It is linearly proportional with the perturbation of the optical fiber, in other words, the alternation of the optical path difference, generated by external perturbation [11]. Thus, precisely demodulating the phase of the reflected lightwave is the key to solve for the distributed perturbation along an optical fiber. A number of demodulation methods have been proposed in the past [12-17]. A. Masoudi and $\mathrm{M}$. Belal used a $3 \times 3$ coupler to measure multiple dynamic perturbations along a $1 \mathrm{~km}$ fiber [16]. Y. Dong proposed a quantitative measurement system using $\mathrm{I} / \mathrm{Q}$ demodulation method [13]. G. Yang developed a vibration sensing technique based on $\Phi-O T D R$ using Hilbert transform, which is able to measure dynamic strain at a frequency as low as $30 \mathrm{~Hz}$ [14].

In this paper, the phase demodulation method, i.e. perturbation measurement method, was inspired by Hilbert transform. The system is based on an UWFBG array assisted $\Phi-O T D R$, and a double-pulse waveform is used as probe signal. The physical spacing between neighboring UWFBGs and the time duration between two pulses are carefully designed, that the reflected optical signal of the front probe pulse overlaps with the reflected optical signal of the rear probe pulse from an adjacent UWFBG. Thus, the two reflected pulses interfere with each other at the receiving side, containing the phase information between two neighboring UWFBGs. So a perturbation on fiber induces the variation of the interference signal from corresponding position. However, due to the fact that the intensity of the interference signal is related to the optical phase change via a trigonometric function, it is hard to demodulate the vibration from the intensity variation of the interference signal as stated in our previous paper [18]. Therefore, a coherent detection scheme is employed in this paper. On the other hand, single probe pulse combined with heterodyne coherent detection cannot locate the perturbation directly from the intensity variation of the signal, because it does not change with the perturbation in this circumstance. 
Thus, one has to demodulate all the phases of the optical signal along the fiber to find the vibration, which severely deteriorate the response speed of the system. Double-pulse can help to locate perturbations easily since it generates an intensity variation to the optical signal at the vibration position. Thus, one only needs to demodulate the phases of the optical signals around the vibration position, which can greatly improve the response speed. In addition, a twice-differential method is proposed to eliminate the noise generated from the laser source, so very low frequency signal can be extracted in experiment.

\section{PRINCIPLE}

The proposed interrogation scheme for UWFBG array is shown in Fig. 1. The UWFBG array has a uniform spatial interval $\Delta \mathrm{L}$ between adjacent UWFBGs. The output of a narrow linewidth laser (NLL) is split into two paths via a 90/10 coupler. The upper path with higher power is intensity modulated to form the probe signal via an acoustic optical modulator (AOM). The probe signal is composed of two optical pulses with time interval of $2 \mathrm{n} \Delta \mathrm{L} / \mathrm{c}$, where $\mathrm{n}$ is the refractive index of fiber and $\mathrm{c}$ is the velocity of light in vacuum, and each pulse has an angular frequency shift of $\Delta \omega$ introduced by the AOM. Therefore, the reflected optical signals of the double-pulse generated from adjacent UWFBGs overlap with each other. The second path with lower power serves as reference path to hold the lightwave property from NLL. The reflected optical signals of the rear and front probe pulses, as well as the reference signal can be described as:

$$
\left\{\begin{array}{l}
\mathbf{E}_{n}=A_{n} \exp \left[-i\left(\omega t+\phi_{n}\right)\right] \\
\mathbf{E}_{n+1}=A_{n+1} \exp \left[-i\left(\omega t+\phi_{n+1}\right)\right] \\
\mathbf{E}_{L o}=A_{L o} \exp \left[-i\left((\omega+\Delta \omega) t+\phi_{L o}\right)\right]
\end{array}\right.
$$

where $A$ represents the magnitude of electrical field, $\omega$ represents the angular frequency of NLL, and $\phi$ represents the phase. The subscript $n$ denotes the reflected signal of the $n^{\text {th }}$ UWFBG, and the subscript Lo denotes the local oscillator signal preserved by the reference path.

Assuming that the reflectivity of the UWFBG array is a constant, when the three signals in (1) are collected and mixed through a $2 \times 150 / 50$ coupler, the ac-coupled intermediate frequency (IF) photocurrent can be described as

$$
I_{n+1} \propto 2 A A_{L o} \cos \left(\frac{\Delta \phi_{n+1}}{2}\right) \cos \left(\Delta \omega t+\phi_{n}-\phi_{L o}+\frac{\Delta \phi_{n+1}}{2}\right)
$$

where $\Delta \phi_{n+1}=\phi_{n+1}-\phi_{n}$ is the phase difference between the front and rear pulses for the $\mathrm{n}^{\text {th }}$ and $(\mathrm{n}+1)^{\text {th }}$ UWFBGs, which can be named as the $(n+1)^{\text {th }}$ UWFBG pair. When the short fiber section within the $(\mathrm{n}+1)^{\text {th }}$ UWFBG pair is disturbed, the phase difference $\Delta \phi_{n+1}$ changes accordingly. From (2) we can see that an external perturbation modulates both phase and amplitude of the ac-coupled IF signal at the receiver, a phase variation $\Delta \phi_{n+1}$ to the IF signal; and the amplitude of the signal via $\cos \left(\Delta \phi_{n+1} / 2\right)$. Thus, the phase change within the target pair is isolated from other pairs in the array. The perturbation within a specific pair can be resolved simply by demodulating the amplitude and phase of the IF signal from the target pair, rather than demodulating all IF signals from all reflectors in a single pulse system.

The Hilbert transform of (2) can be expressed as:

$$
H\left(I_{n+1}\right) \propto 2 A A_{L o} \cos \left(\frac{\Delta \phi_{n+1}}{2}\right) \sin \left(\Delta \omega t+\phi_{n}-\phi_{L o}+\frac{\Delta \phi_{n+1}}{2}\right)
$$

based on (2) and (3), the phase of the $(\mathrm{n}+1)^{\text {th }}$ signal is derived:

$$
\Delta \phi_{n+1}=2\left(\arctan \left(\frac{H\left(I_{n+1}\right)}{I_{n+1}}\right)+m_{n+1} \pi-\Delta \omega t+\phi_{L o}-\phi_{n}\right)
$$

In (4), $m_{n+1} \pi$ ( $m_{n+1}$ is an integer) is an item to unwrap the arctan function, and $\phi_{L o}-\phi_{n}$ is constant ideally. In practical scenario, however, since the item $\cos \left(\Delta \phi_{n+1} / 2\right)$ in (2) varies with perturbation, it induces large error to the result obtained with (4) when $\cos \left(\Delta \phi_{n+1} / 2\right)$ is close to zero. But we can find that $\Delta \phi_{r}$ is constant for the $r^{\text {th }}$ UWFBG pair as long as $r \neq n+1$, thus by rearranging (4), the following equation can be obtained:

$$
\phi_{n+2}=\arctan \left(\frac{H\left(I_{n+2}\right)}{I_{n+2}}\right)+m_{n+2} \pi-\Delta \omega t-\frac{\Delta \phi_{n+2}}{2}+\phi_{L o}
$$

Because the item $\Delta \omega t$ changes with time, the value $\phi_{n+2}$ obtained from (5) is different for different measurement time. This makes it hard to extract the actual phase change induced by external perturbation, so a differential method is implemented. In this method, we also extract the phase $\Delta \phi_{n}$ of the $\mathrm{n}^{\text {th }}$ signal by using (5). Subtracting $\phi_{n}$ from $\phi_{n+2}$ leads to the following equation:

$$
\begin{aligned}
\Delta \phi_{n+2, n}^{\prime}=\phi_{n+2}-\phi_{n}= & 2\left\{\arctan \left[H\left(I_{n+2}\right) / I_{n+2}\right]\right. \\
& \left.-\arctan \left[H\left(I_{n}\right) / I_{n}\right]+C\right\}
\end{aligned}
$$

where $C=\left(m_{n+2}-m_{n}\right) \pi+\left(\Delta \phi_{n}-\Delta \phi_{n+2}\right) / 2$ is constant. Thus, the phase change induced by the perturbation within the $(\mathrm{n}+1)^{\text {th }}$ UWFBG pair is obtained.

\section{EXPERIMENT}

The configuration of experiment is shown in Fig. 1. The length of the sensing fiber was $4.5 \mathrm{~km}$. The whole fiber was integrated with UWFBG array which had equal spatial interval of $50 \mathrm{~m}$. The length of each UWFBG was about $0.05 \mathrm{~mm}$, the central wavelength of all the UWFBGs was $1550 \mathrm{~nm}$ and the bandwidth of the reflective spectrum of the UWFBG was about $5.5 \mathrm{~nm}$. The laser source worked on $1550.12 \mathrm{~nm}$. The output lightwave was split into two paths with a 90:10 optical coupler. The $90 \%$ lightwave was modulated into the double-pulse by an acoustic optical modulator (AOM) and the 10\% lightwave served as reference lightwave. The pulse width of each optical pulse was $300 \mathrm{~ns}$, and the time interval between the two pulses was $500 \mathrm{~ns}$. So the reflected pulses of the front and rear pulses overlapped together. Meanwhile, the probe pulse had $200 \mathrm{MHz}$ frequency shift, induced by the AOM. Then the probe pulse was launched into the sensing fiber through a circulator, and the reflected signal mixed with the reference lightwave. The heterodyne optical signal was received by a photo diode (PD) 


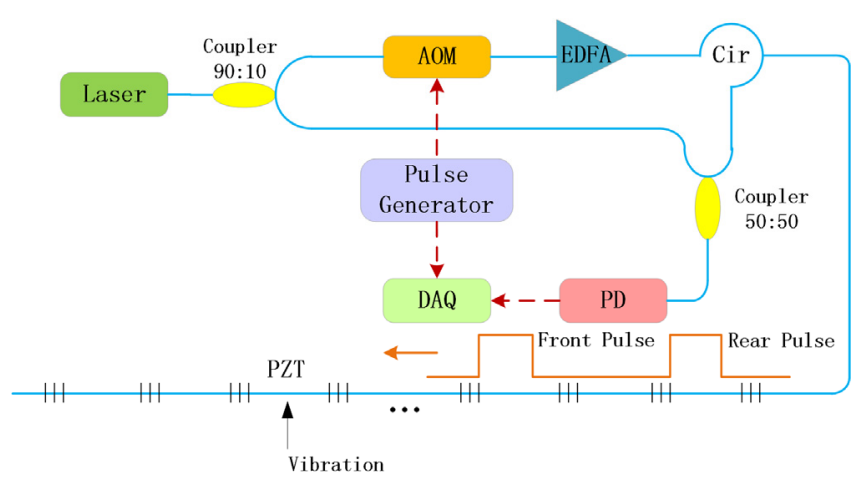

Fig. 1. The experiment setup.

and its output signal was converted to digital form by a data acquisition card (DAQ) with a sampling rate of $500 \mathrm{MSa} / \mathrm{s}$. A PZT vibration source was set at about $4 \mathrm{~km}$.

A $30 \mathrm{~Hz}$ triangular signal was given to drive the PZT. The repetition rate of the double-pulse was $2 \mathrm{kHz}$ and 1000 temporal curves were obtained. The signal obtained from the experiment is shown in Fig. 2, including 50 temporal curves obtained successively. Each peak is the interference result between the reflected front and rear pulses. Its length is determined by the probe pulse width. Obviously, the position of the vibration can be easily located with this method, as shown in the red circle in Fig.2. The two insets in Fig.2 show three temporal curves for $100 \mathrm{~ms}, 200 \mathrm{~ms}$ and $300 \mathrm{~ms}$. The left inset shows the signal at the vibration position. As depicted before, the amplitude of the signal also varies with vibration, thus it induces large error to the extraction of phase when the amplitude is close to zero. Thus, as shown in the right inset in Fig. 2, the signal right after the vibration was chosen to extract the phase according to (5).

Then by applying (5) to the signal at the vibration position and that right before it, the phase variation induced by the vibration was obtained. The demodulated result is show in Fig. 3 , where the black curve represents the driving signal applied on the PZT and the read and blue ones are the demodulated phase signal with $5 \mathrm{Vpp}$ and $10 \mathrm{Vpp}$ driving voltages respectively. Apparently, the demodulated phase restores the driving signal accurately.

Next, we changed the driving signal to sinusoidal waveform. The demodulated phase result and its spectrum are shown in Fig. 4. Synthesizing Fig. 3 and Fig. 4 we can see that the

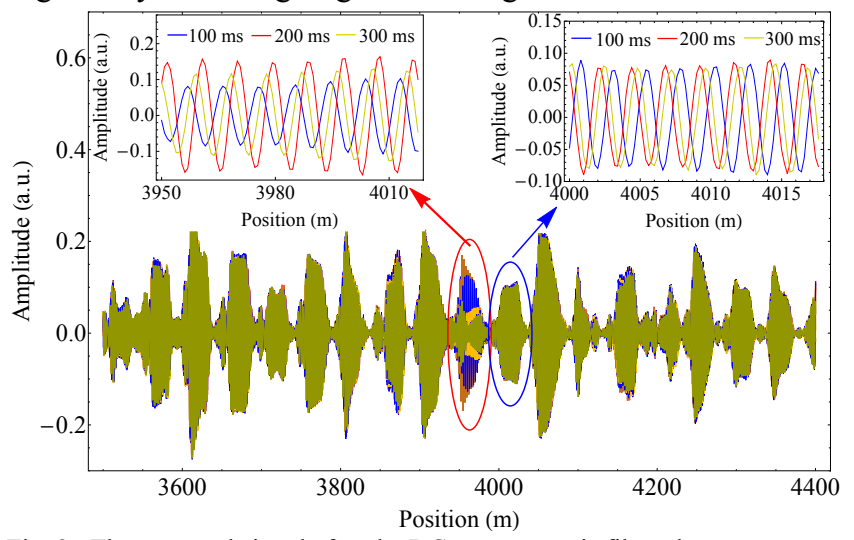

Fig. 2. The temporal signal after the DC component is filtered.

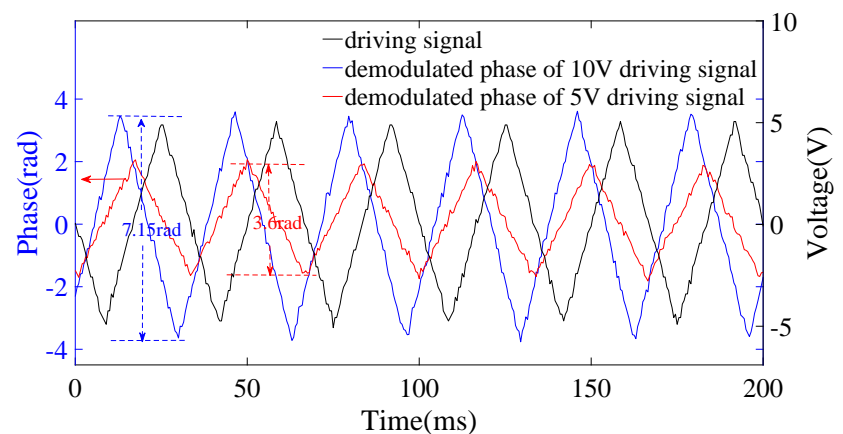

Fig. 3. The comparison of the demodulated phase signal and the driving electric signal.
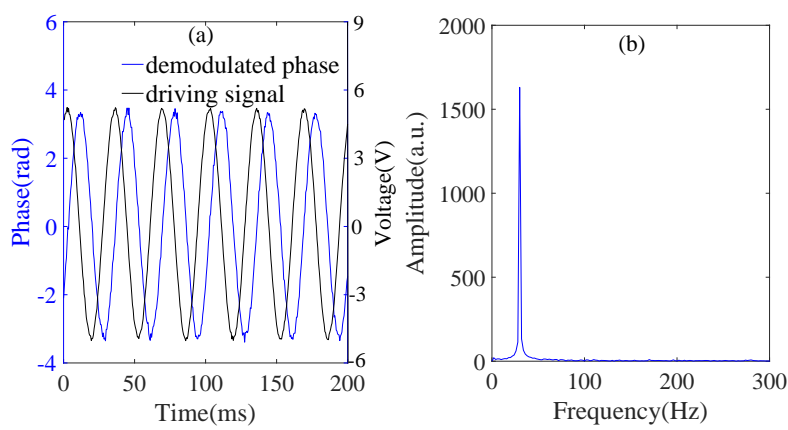

Fig. 4. The demodulated result for (a) sinusoidal driving signal and (b) its frequency spectrum.

method can demodulate different signal waveforms effectively. Then the driving voltage varied from $2 \mathrm{Vpp}$ to $10 \mathrm{Vpp}$ with a step of $0.5 \mathrm{Vpp}$ and the amplitudes of the fundamental frequency in the spectrum were recorded. In this experiment, the signal under each driving voltage was measured ten times. The mean value of the results and the driving voltage are shown in Fig. 6, where a good linear relationship $\left(\mathrm{r}^{2}=0.9998\right)$ was observed. The maximum standard deviation of the FFT amplitude for each driving voltage is 17.14 , which determines the minimum detectable phase difference is $0.068 \mathrm{rad}$, corresponding to a fiber length variation of $14.85 \mathrm{~nm}$ [19].

Furthermore, the ability of measuring very low frequency with the proposed method was also demonstrated. In this experiment, the sinusoidal driving signal was set at $0.2 \mathrm{~Hz}$ and $10 \mathrm{Vpp}$. The repetition rate of the double-pulse was changed to $100 \mathrm{~Hz}$, to match the slow vibration. Then 1000 temporal curves were obtained which would cover two periods of the

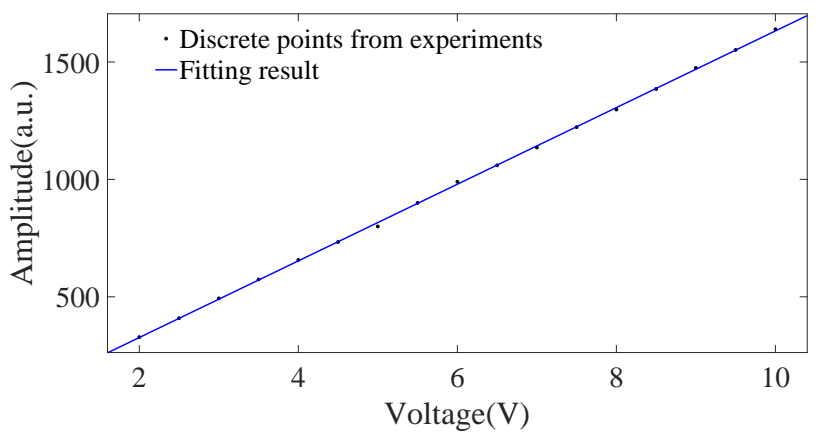

Fig. 5. The relationship between the driving voltage and the amplitude of the frequency spectrum of the demodulated phase result. 

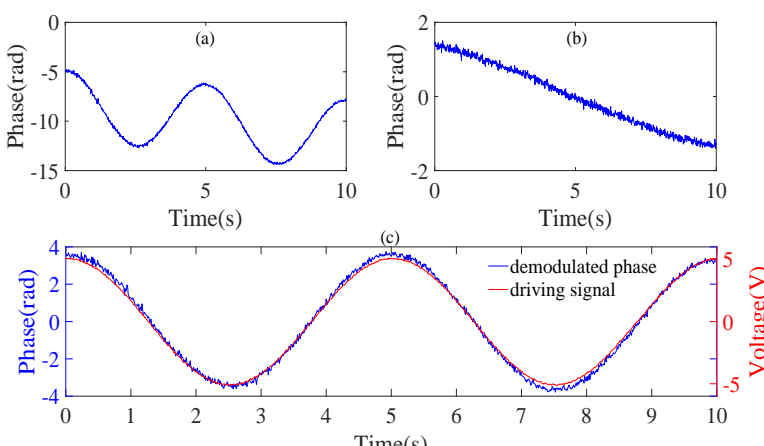

Fig. 6. The process of demodulating $0.2 \mathrm{~Hz}$ sinusoidal signal. (a)The primary result mixed with the influence of frequency drift. (b)The phase drift caused by frequency drift obtained through the differential method. (c) The final demodulated result.

vibration. The demodulated result is shown in Fig. 6 .

In Fig. 6(a), the $0.2 \mathrm{~Hz}$ signal can be observed clearly, but the curve also shows a very slow downward trend with time. We cannot determine whether this is induced by an actual signal or by a noise. Although we had implemented a differential operation between $\Delta \phi_{n+1}$ and $\Delta \phi_{n}$, which eliminated most common noises before the $(\mathrm{n}-1)^{\text {th }}$ UWFBG, there is still $100 \mathrm{~m}$ fiber length difference between the $(\mathrm{n}+1)^{\text {th }}$ and $(\mathrm{n}-1)^{\text {th }}$ UWFBGs. The frequency drift of the laser source on this fiber section is non-negligible during a long measuring time. Thus we proposed a twice-differential method to further reduce the influence of the laser frequency drift. In this method, the differential operation by using (5) was implemented to the signals between another two UWFBG pairs, whose interval was also $100 \mathrm{~m}$ and between which there was no perturbation. The result is shown in Fig. 6(b), where also exhibits a downward tread signal. Finally, by subtracting the result in Fig. 6(b) from that of Fig.6(a), the influence of the laser source on the $100 \mathrm{~m}$ fiber section can also be removed. The final result is shown in Fig.6(c). By comparing with the driving signal, they are totally coincident.

The reflectivity of the UWFBG used in experiment was about $-40 \mathrm{~dB}$. So the power of the reflected signal for a probe pulse with $300 \mathrm{~ns}$ pulse width is about $18 \mathrm{~dB}$ higher than that of the Rayleigh backscattering. Taking the advantage of high SNR, the sensitivity of the system was very good. According to the preceding signal processing procedure, in order to demodulate the phase of a given position and to eliminate the influence of laser frequency drift, the signals between four UWFBG pairs' phase information were calculated, costing $0.66 \mathrm{~s}$ in the experiment. If double-pulse was not adopted, however, the vibration position cannot be located directly, so the phase information of all the signals along the fiber should be demodulated. There were total 88 UWFBGs in the fiber of the experiment, it will cost $14.52 \mathrm{~s}$ to process all the UWFBGs' signal. Therefore, using double-pulse can improve the response speed significantly.

\section{CONCLUSION}

In conclusion, we demonstrate a phase demodulation method based on UWFBG array assisted $\Phi$-OTDR. We use double-pulse to locate the vibration and use heterodyne coherent detection and Hilbert transform to quantitatively demodulate the phase variation of the lightwave induced by vibration. Both sinusoidal signal and triangle signal are demodulated accurately. The demodulated result has a very linear relationship with the amplitude of the driving signal and the minimum detectable fiber length variation is $14.85 \mathrm{~nm}$. Because of the high SNR of the reflected signal and the twice-differential method, very low frequency of vibration can be detected. A $0.2 \mathrm{~Hz}$ sinusoidal driving signal is properly detected.

\section{REFERENCES}

[1] J. C. Juarez, E. W. Maier, C. Kyoo Nam and H. F. Taylor, "Distributed fiber-optic intrusion sensor system," J. Lightwave Technol., vol. 23, no. 6, pp.2081-2087, 2005 .

[2] Y. Lu, T. Zhu, L. Chen and X. Bao, "Distributed Vibration Sensor Based on Coherent Detection of Phase-OTDR," J. Lightwave Technol., vol. 28, no. 22, pp.3243-3249, 2010 .

[3] L. Zhou, F. Wang, X. Wang, Y. Pan, Z. Sun, J. Hua and X. Zhang, "Distributed Strain and Vibration Sensing System Based on 1884-1887, 1884-1887, 2015.

[4] Z. Wang, Z. Pan, Q. Ye, B. Lu, Z. Fang, H. Cai and R. Qu, "Novel distributed passive vehicle tracking technology using phase sensitive optical time domain reflectometer," Chin. Opt. Lett., vol. 13, no. 10, pp. 30-34, 2015.

[5] X. Bao and L. Chen, "Recent progress in distributed fiber optic sensors," Sensors(Basel), vol. 12, no. 7, pp.8601-8639, 2012.

[6] Y. Shi, H. Feng and Z. Zeng, "A long distance phase-sensitive optical time domain reflectometry with simple structure and high locating accuracy," Sensors(Basel), vol. 15, no. 9, pp.21957-21970, 2015.

[7] F. Peng, N. Duan, Y. J. Rao and J. Li, "Real-time position and speed monitoring of trains using phase-sensitive OTDR," IEEE Photon. Technol. Lett., vol. 26, no. 20, pp.2055-2057, 2014.

[8] C. Wang, Y. Shang, X.H. Liu, H.H. Yu, D.S. Jiang and G.D. Peng. "Distributed OTDR-interferometric sensing network with identical ultra-weak fiber Bragg gratings," Opt. Express., vol. 23, no. 22, pp. 29038-29046, 2015.

[9] P. Han, Z. Li, L. Chen, X. Bao, "A High-Speed Distributed Ultra-Weak FBG Sensing System With High Resolution;" IEEE Photonics Technology Letters, vol. 29, no. 15, pp. 1249-1252, 2017

[10] L. Ma, C. Ma, Y. Wang, D. Y. Wang, A. Wang, "High-Speed Distributed Sensing Based on Ultra Weak FBGs and Chromatic Dispersion;" IEEE Photonics Technology Letters, vol. 28, no. 12, pp. 1344-1347, 2016.

[11] Namihira and Yoshinori. "Opto-elastic constant in single mode optical fibers." J. Lightwave Technol., vol.3, no.5 pp. 1078-1083, 1985.

[12] A. Masoudi, M. Belal and T. P. Newson. "A distributed optical fibre dynamic strain sensor based on phase-OTDR." Meas. Sci. Technol., vol. 24, no.8, p. 085204, 2013.

[13] Y. Dong, X. Chen, E. Liu, C. Fu, H. Zhang and Z. Lu. "Quantitative measurement of dynamic nanostrain based on a phase-sensitive optical time domain reflectometer." Appl. Opt. vol. 55, no.28, pp.7810-7815, 2016.

[14] G. Yang, X. Fan, S. Wang, B. Wang, Q. Liu and Z. He, "Long-Range Phase-Sensitive OTDR." IEEE Photonics J, vol. 8, no 3 pp.1-12, From

[15] Z. Sha, H. Feng and Z. Zeng, "Phase demodulation method in phase-sensitive OTDR without coherent detection," Opt. Express., vol. 25, no. 5, pp. 4831-4844, 2017

[16] M.G. Taylor, "Phase estimation methods for optical coherent detection using digital signal processing," J. Lightwave Technol., vol. 27, no. 7, pp.901-914, 2009.

[17] C. Wang, Y. Shang, X. Liu and G. Peng, "Distributed acoustic mapping based on interferometry of phase optical time-domain reflectometry, Opt. Commun., vol. 346, pp.172-177, 2015.

[18] T. Liu, F. Wang, X. Zhang, L. Zhang, Q. Yuan, Y. Liu and Z.Yan. "Phase sensitive distributed vibration sensing based on ultraweak fiber Bragg grating array using double-pulse." Opt. Eng., vol. 56, no.8: $084104,2017$.

[19] Z. Fang, G. Qin, R. Qu, H.Cai,"Fiber sensitivity and fiber optic devices" in Fundamentals of Optical Fiber Sensors, China, 2013, pp. 58-59. 